\title{
Evaluation of pupil diameter after posterior chamber phakic intraocular lens implantation
}

\author{
Abstract \\ Purpose To observe the changes of pupil size \\ over time after Visian Implantable Collamer \\ Lens (ICL) implantation. \\ Patients and methods We retrospectively \\ examined 30 eyes of 23 consecutive patients \\ undergoing ICL implantation. We measured \\ the entrance and real pupil diameters using a \\ Hartmann-Shack aberrometer (KR-9000, \\ Topcon, Tokyo, Japan) before and 1 day, \\ 1 week, and 1, 3, 6, and 12 months after \\ surgery. We also investigated its relationship \\ with the amount of vaulting using slit-lamp \\ microscopy 1 year postoperatively. \\ Results The entrance pupil diameters were \\ $6.24 \pm 0.66$ (mean \pm standard deviation) $\mathrm{mm}$ \\ preoperatively, and $5.53 \pm 0.69,6.18 \pm 0.61$, \\ $6.21 \pm 0.80,6.29 \pm 0.74,6.23 \pm 0.76$, and \\ $6.40 \pm 0.70 \mathrm{~mm}, 1$ day, 1 week, and 1, 3, 6, \\ and 12 months postoperatively, respectively; \\ and the respective real pupil diameters \\ were $5.44 \pm 0.55 \mathrm{~mm}$ preoperatively, and \\ $4.95 \pm 0.60,5.53 \pm 0.52,5.55 \pm 0.69,5.63 \pm 0.64$, \\ $5.57 \pm 0.64$, and $5.72 \pm 0.60 \mathrm{~mm}, 1$ day, 1 week, \\ and 1, 3, 6, and 12 months postoperatively. \\ Pupil diameters and the amount of vaulting \\ were not significantly associated (Pearson \\ correlation coefficient $r=0.14, P=0.45$ for \\ entrance pupil, $r=0.13, P=0.49$ for real pupil). \\ Conclusions Both pupil diameters decreased \\ transiently 1 day after ICL implantation, but \\ soon recovered to the preoperative level, \\ indicating that intraoperative mechanical \\ irritation of the uveal tissue and the early \\ postoperative inflammatory response may \\ induce transient decreases in pupil diameter, \\ and that this surgical technique alone with \\ appropriate ICL size selection probably \\ induces no significant pupil diameter change. \\ Eye (2010) 24, 588-594; doi:10.1038/eye.2009.170; \\ published online 10 July 2009
}

${ }^{1}$ Department of Ophthalmology, University of Kitasato School of Medicine, Kanagawa, Japan

${ }^{2}$ School of Allied Health Sciences, University of Kitasato, Kanagawa, Japan

Correspondence: K Kamiya, Department of

Ophthalmology,

University of Kitasato School of Medicine,

1-15-1 Kitasato, Sagamihara,

Kanagawa 228-8555, Japan

Tel: +8142778 9012;

Fax: +81427789920

E-mail: kamiyak-tky@

umin.ac.jp

Received: 30 December 2008

Accepted in revised form: 22 May 2009

Published online: 10 July 2009
K Kamiya ${ }^{1}$, K Shimizu ${ }^{1}$, A Igarashi ${ }^{1}$ and $\mathrm{H}$ Ishikawa ${ }^{2}$

Keywords: ICL; phakic IOL; pupil; vaulting

Introduction

The Visian Implantable Collamer Lens (ICL, STAAR Surgical, Nidau, Switzerland), a posterior chamber phakic intraocular lens (IOL), has become widely accepted in recent years as an effective means of correcting moderate to high ametropia. ${ }^{1-10}$ This surgical procedure may have advantages over laser in situ keratomileusis (LASIK) because it is safer and more effective, and provides highly predictable and stable results, especially in the correction of high myopia. Moreover, the lens is removable and replaceable with another ICL, whereas, with LASIK, this cannot be done even when unexpected refractive outcomes occur after surgery. In addition, the toric ICL has been shown in recent years to be effective for the correction of high myopic astigmatism. ${ }^{11-14}$

Pupil diameter has an important function in the refractive outcomes of the surgical procedure. Considering that the optic of an ICL needs to be secured in contact with the back surface of the iris because of the anterior vault design of the ICL, it is possible that the pupil diameter may be altered by ICL fixation. However, the changes in pupil size, especially in real pupil size, in ICL-implanted eyes have not been fully elucidated so far. Moreover, the relationship of the pupil diameter with the amount of vaulting (the distance between the posterior surface of the ICL and the anterior surface of the crystalline lens), which is also important for the assessment of the safety of this surgical procedure, has not been investigated. The purpose of the current study is to longitudinally assess the pupil diameter in 
ICL-implanted eyes, and to assess its relationship with the vaulting in such eyes.

\section{Materials and methods}

Thirty eyes of the 23 consecutive patients ( 5 men and 18 women) who underwent implantation of the posterior phakic ICL (STAAR Surgical) for the correction of myopia, and who returned regularly for postoperative examination, were included in this retrospective study. The age of patients at the time of surgery was $35.7 \pm 12.0$ years (mean age \pm standard deviation (SD); range: from 23 to 46 years old). The preoperative refraction was $-10.10 \pm 2.90$ diopters (D) (range: from -4.00 to $-15.00 \mathrm{D})$. Informed consent was obtained from all patients. The study adhered to the tenets of the Declaration of Helsinki. Institutional Review Board approval was not required for this retrospective study.

Lens power calculations were performed by the manufacturer (STAAR Surgical) using a modified vertex formula. The size of the ICL was chosen by the manufacturer on the basis of the horizontal corneal diameter and anterior chamber depth from the corneal endothelium measured with a scanning-slit topograph (Orbscan IIz, Bausch and Lomb, Rochester, USA).

Preoperatively, the patients underwent peripheral iridectomies at two sites with a neodymium-YAG laser. On the day of surgery, the patients were administered dilating and cycloplegic agents. After topical anaesthesia, a model V4 ICL was inserted through a 3-mm clear corneal incision with the use of an injector cartridge (STAAR Surgical) after placement of viscoelastic material (Opegan Santen, Osaka, Japan) into the anterior chamber. After the ICL had been placed in the posterior chamber, the remaining viscoelastic material was completely washed out of the anterior chamber with balanced salt solution and then a miotic agent (acetylcholine chloride, Ovisort Daiichi-Sankyo, Tokyo, Japan) was instilled. All surgeries were uneventful and no proven intraoperative complication was observed. After surgery, steroidal ( $0.1 \%$ betamethasone, Rinderon Shionogi, Osaka, Japan) and antibiotic (levofloxacin, Cravit Santen, Osaka, Japan) medications were topically administered four times daily for 2 weeks, after which the dose was steadily reduced.

The entrance pupil diameter was evaluated with a Hartmann-Shack aberrometer (KR-9000, Topcon, Tokyo, Japan) under low-light conditions (10 lux) with monocular vision. The patients were evaluated in a silent, darkened room with illumination. After $3 \mathrm{~min}$ dark adaptation, we carried out this measurement three times at the same time of day under the same conditions as those in which the patients were resting to reduce the individual changes in pupil diameter. All examinations were performed by two experienced ophthalmic technicians. Video images were captured, and the entrance pupil diameter was measured automatically with digital infrared pupillometry. The average values of the three consecutive measurements were used for statistical analysis. We also calculated the real pupil diameter using the following formula: ${ }^{15}$ the real pupil diameter $(\mathrm{mm})=$ the entrance pupil diameter $(\mathrm{mm}) \times(1-A K / 1.3375)$, where $A$ is the anterior chamber depth $(\mathrm{m}), K$ is the central corneal refractive power (D), and 1.3375 is the standard refractive index of the cornea. In this study, anterior chamber depth was determined as the distance between the corneal epithelium and the anterior surface of the implanted ICL at each postoperative visit using the scanning-slit topograph to calculate the real pupil diameter. For each eye, we hand picked the appropriate image and examined it in detail to ensure that the anterior surface of the ICL was correctly identified. The mean keratometric readings were measured using an autorefractometer (ARK-700A, Nidek, Gamagori, Japan). To assess the amount of vaulting, an anterior-segment image was obtained using slit-lamp microscopy 1 year postoperatively in accordance with the procedures reported by Gonvers et al. ${ }^{16-18}$ In brief, the digitized photographs were taken after pupil dilation using a slit-lamp camera with the beam as thin as possible and focused on the centre of the ICL. Using the NIH-Image analysis software program, we calculated the central vaulting of the ICL over the crystalline lens (the distance between the posterior surface of the ICL and the anterior surface of the crystalline lens) to compare it with the central thickness of the ICL, which was provided by the ICL manufacturer (STAAR Surgical). In addition, to assess the repeatability of the measurement, 16 eyes of 16 normal volunteers underwent three consecutive measurements of the entrance pupil diameter with this device at 1-week intervals.

All statistical analyses were performed using SPSS (SPSS Inc., Chicago, USA). The results are expressed as mean $\pm \mathrm{SD}$, and a value of $P<0.05$ was considered statistically significant.

\section{Results}

Patient demographics are summarized in Table 1. The time courses of the entrance and real pupil diameters are shown in Figure 1. The entrance pupil diameters were $6.24 \pm 0.66$ (mean \pm SD) $\mathrm{mm}$ preoperatively and $5.53 \pm 0.69,6.18 \pm 0.61,6.21 \pm 0.80,6.29 \pm 0.74,6.23 \pm 0.76$, and $6.40 \pm 0.70 \mathrm{~mm}, 1$ day, 1 week, and 1, 3, 6, and 12 months postoperatively, respectively. The real pupil diameters were $5.44 \pm 0.55 \mathrm{~mm}$ preoperatively and $4.95 \pm 0.60,5.53 \pm 0.52,5.55 \pm 0.69,5.63 \pm 0.64,5.57 \pm 0.64$, and $5.72 \pm 0.60 \mathrm{~mm}, 1$ day, 1 week, and 1, 3, 6, and 12 
Table 1 Demographic data of the study population

Patient demographics

Age (years)

Gender (\% female)

Manifest spherical equivalent (D)

Manifest cylinder (D)

Central cornea thickness $(\mu \mathrm{m})$

Mean keratometric readings (D)

White to white distance (mm)

Anterior chamber depth (from the corneal epithelium) (mm)
$35.7 \pm 12.0$ years (range, $23-46$ years)

$78 \%$

$-10.10 \pm 2.90 \mathrm{D}$ (range, -4.00 to $-15.00 \mathrm{D}$ )

$0.99 \pm 0.65 \mathrm{D}$ (range, $0.00-4.00 \mathrm{D}$ )

$543.8 \pm 29.5 \mu \mathrm{m}$ (range, $492-639 \mu \mathrm{m}$ )

$44.6 \pm 1.1 \mathrm{D}$ (range, $41.8-46.4 \mathrm{D}$ )

$11.5 \pm 0.3 \mathrm{~mm}$ (range, $11.0-12.1 \mathrm{~mm}$ )

$3.80 \pm 0.34 \mathrm{~mm}$ (range, $3.35-4.64 \mathrm{~mm}$ )

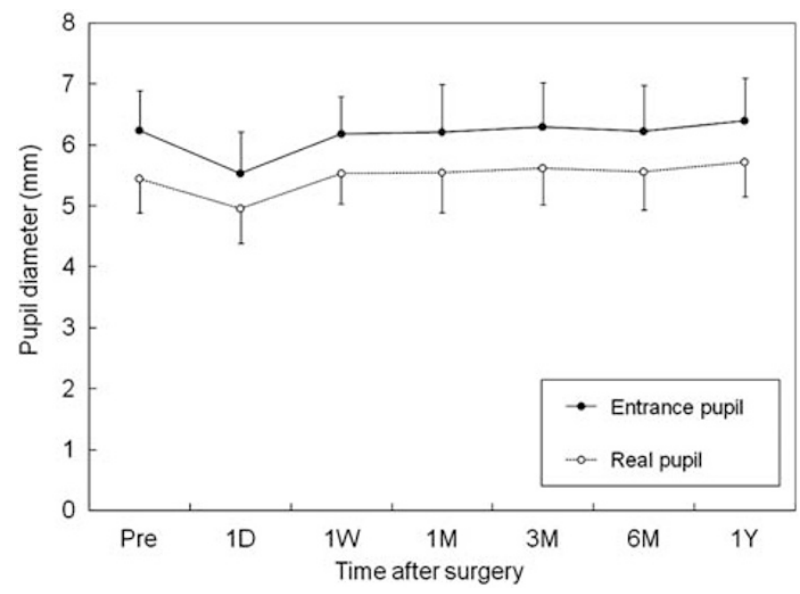

Figure 1 Time course of the entrance and real pupil diameters after ICL implantation. Pupil diameter may transiently decrease at 1 day after ICL implantation, but this decrease returns soon to the preoperative levels, and then stabilizes thereafter. The variance of the data was statistically significant $(P<0.001$ for both entrance and real pupil diameters, repeated-measures analysis of variance). Multiple comparisons showed significant differences between measurements for entrance and real pupil diameters made before surgery and 1 day after, but no significant differences between measurements made before surgery and (a) 1 week after, (b) 1 month after, (c) 3 months after, (d) 6 months after, or (e) 12 months after. D, day; W, week; $\mathrm{M}$, month; $\mathrm{Y}$, year; bar represents $\mathrm{SD}$.

months postoperatively, respectively. Changes in entrance pupil diameter from before surgery to 1 day after, from 1 day to 1 week after, from 1 week to 1 month after, from 1 to 3 months after, from 3 to 6 months after, and from 6 months to 1 year after were $-0.71 \pm 0.58$, $0.65 \pm 0.46,0.02 \pm 0.51,0.08 \pm 0.56,-0.06 \pm 0.57$, and $0.17 \pm 0.65 \mathrm{~mm}$, respectively. The respective changes in real pupil diameter from before surgery to 1 day after, from 1 day to 1 week after, from 1 week to 1 month after, from 1 to 3 months after, from 3 to 6 months after, and from 6 months to 1 year after were $-0.49 \pm 0.54$, $0.58 \pm 0.41,0.02 \pm 0.46,0.08 \pm 0.51,-0.06 \pm 0.51$, and $0.15 \pm 0.58 \mathrm{~mm}$. The variance of the data was statistically significant $(P<0.001$ for both entrance and real pupil

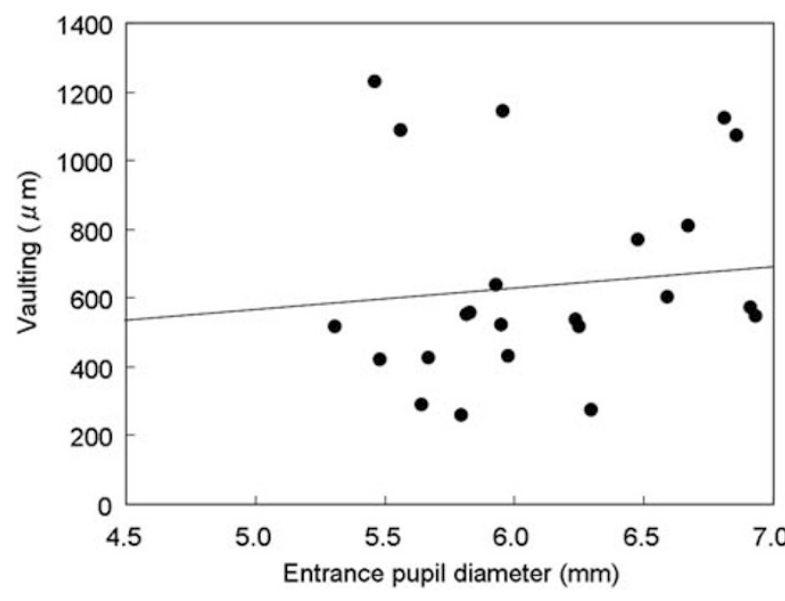

Figure 2 A scatter plot showing no significant correlation between the entrance pupil diameter and the amount of vaulting 1 year after ICL implantation (Pearson correlation coefficient $r=0.14, P=0.45$ ).

diameters, repeated-measures analysis of variance). Multiple comparisons showed significant differences between measurements for entrance pupil diameter made before surgery and 1 day after $(P<0.001$, Dunnett Test), but no significant differences between measurements made before surgery and (a) 1 week after ( $P=1.00)$, (b) 1 month after $(P=1.00)$, (c) 3 months after $(P=1.00)$, (d) 6 months after $(P=1.00)$, or (e) 12 months after $(P=0.90)$. They also showed significant differences between measurements for real pupil diameter made before surgery and 1 day after $(P=0.01)$, but no significant differences between measurements made before surgery and (a) 1 week after $(P=0.99)$, (b) 1 month after $(P=0.96)$, (c) 3 months after $(P=0.71)$, (d) 6 months after $(P=0.93)$, or (e) 12 months after $(P=0.30)$.

The central vaulting of the ICL 1 year postoperatively was $661.8 \pm 299.9 \mu \mathrm{m}$ (range: from 260 to $1230 \mu \mathrm{m}$ ). We found no significant correlation between the two pupil diameters and the amount of vaulting 1 year after ICL implantation (Pearson correlation coefficients: $r=0.14$, $P=0.45$ for entrance pupil (Figure 2); $r=0.13, P=0.49$ for real pupil (Figure 3)). Neither pupillary block nor contact 


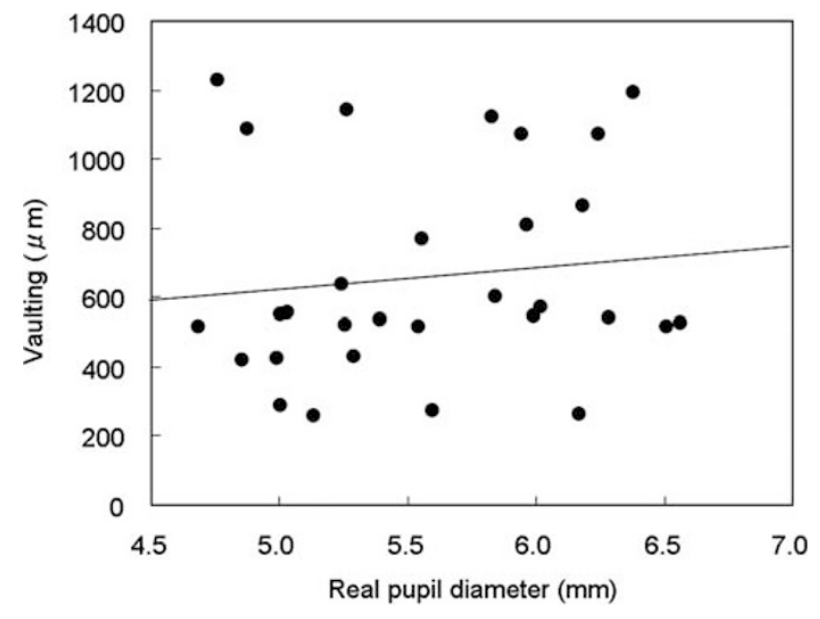

Figure 3 A scatter plot showing no significant correlation between the real pupil diameter and the amount of vaulting 1 year after ICL implantation (Pearson correlation coefficient $r=0.13, P=0.49$ ).

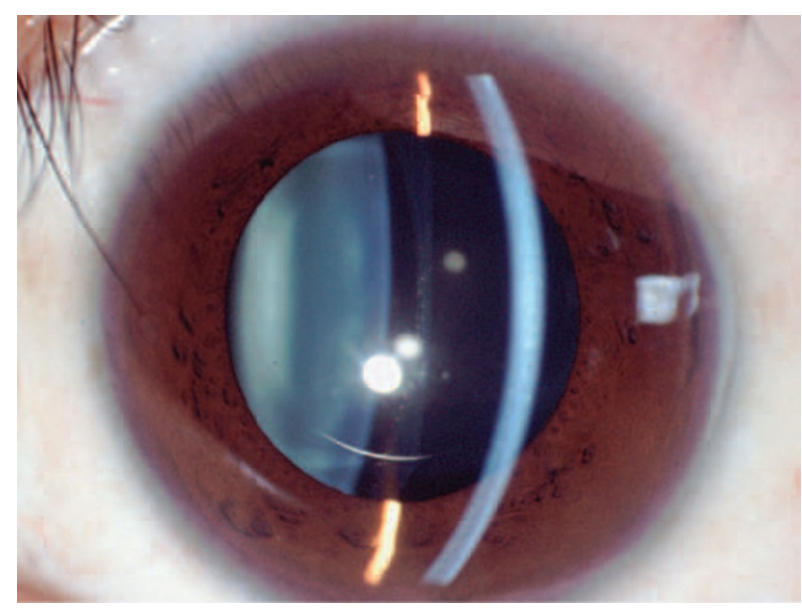

Figure 4 Slit-lamp photograph of the eye undergoing ICL implantation.

between the ICL and the crystalline lens was observed in any case throughout the observation period (Figure 4).

The mean difference between the three consecutive measurements of the entrance pupil size with this aberrometry $( \pm 95 \%$ LoA) was $0.05 \pm 0.16 \mathrm{~mm}$ $(-0.27$ to $0.38 \mathrm{~mm})$.

\section{Discussion}

In this study, our results showed that the entrance diameter of the pupil decreased transiently at 1 day after ICL implantation, which soon increased to the preoperative levels at 1 week after implantation and stabilized thereafter. Considering that the myotic agent (acetylcholine chloride) was considered to work within several hours in a clinical setting, and that the clinically apparent inflammation was seen only 1 day after surgery, the transient reduction of pupil size may be attributed to intraoperative mechanical irritation of the uveal tissue and the early postoperative inflammatory response, and ICL implantation alone does not induce a significant change in pupil diameter. The transient decreases indicate that visual performance, such as visual acuity or higher-order aberrations compromising contrast sensitivity at 1 day after surgery, may be influenced by pupil diameters smaller than those before surgery. ${ }^{19}$ The return to the preoperative levels indicates that this surgical technique itself has negligible effects on pupil size, even though it requires contact between the optic of the ICL and the back surface of the iris. On the other hand, Keuch and Bleckmann ${ }^{19}$ reported that the rate of pupil contraction and redilation, the pupil diameter, and the amplitude of pupil constriction were all reduced after surgery, suggesting that an ICL can cause mechanical irritation of the pupil, resulting in constriction and redilation. Mechanical irritation of the uveal tissue may have played a role in the postoperative pupil reaction. Chun et $a l^{20}$ also reported that, using a scanning-slit topograph, there was a significant decrease in pupil diameter 1 and 3 months postoperatively, and that the diameter returned to the postoperative levels at 6 and 12 months, suggesting that mechanical contact and rubbing between the ICL and the posterior iris surface irritated the pupil and decreased the pupil diameter. At present, we have no clear explanations for this discrepancy. However, detailed measurement conditions such as the brightness of the room, the number of times measurements were carried out, or the time of day at which they were made, as well as the condition of the patient were not fully described in their manuscripts, and thus it is still unclear whether ICL implantation induces a significant change in pupil size because the differences of these conditions are possible sources of measurement error. Moreover, as the ICL optic needs to be secured in contact with the posterior iris surface because of the anterior vault design of the ICL, the pupil size may be enlarged by ICL fixation, especially when the amount of vaulting is large, possibly as a result of the larger ICL size selection. ${ }^{18}$ It is suggested that an appropriate ICL size selection may also be an important factor in determining the postoperative pupil diameter in ICL-implanted eyes. Petternel et $a l^{21}$ showed that the vaulting was significantly reduced under photopic conditions with constriction of the pupil after ICL implantation, but that no significant changes in the vaulting were observed during subjective accommodation or after application of pilocarpine. In this study, we also showed that the amount of vaulting 
is not significantly associated with the pupil size in ICL-implanted eyes. In addition, no contact or high vaulting in excess of $1.25 \mathrm{~mm}$ between the ICL and the crystalline lens was observed in any case during the 1-year follow-up period, suggesting that no extreme underestimation or overestimation of ICL size occurred. We consider that uncomplicated ICL implantation, taken together with an appropriate ICL size selection, does not induce a significant change in pupil diameter. Recently, the rotating Scheimpflug imaging system (Pentacam Oculus), ${ }^{16,22}$ and anterior-segment optical coherence tomograph (Visante OCT Carl Zeiss Meditec, Dublin, CA, USA) ${ }^{23,24}$ provided highly accurate and reproducible measurements of anterior-segment biometry and thus become more helpful in evaluating the relationship of the ICL with the pupil metrics. A further study is necessary to confirm the authenticity of the results.

It is known that the entrance pupil observed in a clinical setting is a virtual image of the anatomical pupil. The entrance pupil is magnified and displaced anteriorly by the refractive power of the cornea. Accordingly, we also calculated the diameter of the real pupil adjusted by the anterior chamber depth and the central corneal refractive power, and obtained similar results: the real diameter of the pupil decreased transiently at 1 day after ICL implantation, but soon increased to the preoperative levels at 1 week after implantation and stabilized thereafter.

It has been shown that the pupil size can be influenced not only by the patient background, for example by age, ${ }^{25-31}$ manifest refraction, ${ }^{32}$ and the accommodative state of the eye, ${ }^{33,34}$ and by various sensory and emotional conditions, ${ }^{35}$ but also by measurement conditions affecting the level of retinal illuminance. ${ }^{36,37}$ This measurement does not necessarily offer high reproducibility. Although we accept that the measurements of pupil diameter in ICL-implanted eyes are better than those in normal eyes, it is still difficult to assess pupil diameter at 1-week intervals in patients undergoing ICL implantation with this device. In our study, the mean difference between three consecutive measurements of pupil diameter with this device $( \pm 95 \%$ LoA) was $0.05 \pm 0.16 \mathrm{~mm}(-0.27$ to $0.38 \mathrm{~mm})$ in normal eyes. Although small test-retest variability does not necessarily mean high accuracy, it is related to the reproducibility of the measurements for elucidating the applicability of data. Hence, we believe that this device offers reasonable accuracy in the measurement of the changes in the pupil diameter over time.

There are several limitations to this study. First, we measured the pupil diameter under mesopic conditions (10 lux) with monocular vision, and so our measurements in this study do not accurately reflect the natural binocular viewing conditions of the patients.
However, these natural viewing conditions may include not only the photopic, but also the mesopic conditions under which we assessed the pupil size. We are currently conducting a further study to assess pupil size under natural viewing conditions without occlusion. Second, we did not quantitatively assess long-term inflammatory responses, which are a possible source of changes in pupil size ${ }^{38,39}$ after ICL implantation. It has been shown that aqueous flare increased by $49 \%$ in the first postoperative month, and decreased afterward, but then remained above preoperative values for the entire 2-year follow-up period. ${ }^{40}$ In this study, we found no clinically demonstrable inflammation during the follow-up period, except on the first postoperative day. In other preliminary data of ours, aqueous flare significantly increased 1 day postoperatively, but recovered soon, returning to within the range of normal values 1 month postoperatively, and stabilized thereafter (unpublished data). Therefore, we assume that the effects of postoperative inflammation on pupil metrics are negligible, at least in the late postoperative period. Third, we measured the central vaulting of the ICL when the pupil was dilated and determined its diameter only at 1 year after surgery in this study. As the ICL optic remains in contact with the rear surface of the iris, the iris may push the ICL towards the crystalline lens before mydriasis. Moreover, the anterior surface of the crystalline lens was shifted posteriorly after mydriasis. Accordingly, pupil dilation appears to increase the amount of the vaulting of the ICL. In our preliminary data, pupil dilation tends to slightly increase the amount of vaulting, but this increase between pre- and postmydriasis remains constant (unpublished data). In addition, it has been reported that the vaulting showed a tendency to decrease slightly over time, ${ }^{16,41}$ suggesting that ICL collamer material may not have a permanent memory. Further studies with longer follow-ups are required for longitudinal assessment of the behaviour of the ICL.

In conclusion, our results support the view that pupil diameter may transiently decrease at 1 day after ICL implantation, but returns soon to the preoperative levels, and stabilizes thereafter. The transient reduction of the pupil diameter may be caused by intraoperative mechanical irritation of the uveal tissue and the early postoperative inflammation, and uncomplicated ICL implantation with an appropriate ICL size selection may not induce a significant change in either the entrance or the real pupil size. There was no significant association between pupil size and the amount of vaulting in ICLimplanted eyes when an appropriate ICL size is selected. More prolonged and careful observation with a large number of patients is necessary for clarification of these aspects. 


\section{Acknowledgements}

Dr Shimizu is a consultant to STAAR Surgical. The remaining authors have no commercial or proprietary in the materials presented therein.

\section{References}

1 Zaldivar R, Davidorf JM, Oscherow S. Posterior chamber phakic intraocular lens for myopia of 8 to 19 diopters. J Refract Surg 1998; 14: 294-305.

2 Sanders DR, Brown DC, Martin RG, Shepherd J, Deitz MR, DeLuca M. Implantable contact lens for moderate to high myopia: phase 1 FDA clinical study with 6-month followup. J Cataract Refract Surg 1998; 24: 607-611.

3 Jimenez-Alfaro I, Gomez-Telleria G, Bueno JL, Puy P. Contrast sensitivity after posterior chamber phakic intraocular lens implantation for high myopia. J Refract Surg 2001; 17: 641-645.

4 Uusitalo RJ, Aine E, Sen NH, Laatikainen L. Implantable contact lens for high myopia. J Cataract Refract Surg 2002; 28 29-36.

5 Bloomenstein MR, Dulaney DD, Barnet RW, Perkins SA. Posterior chamber phakic intraocular lens for moderate myopia and hyperopia. Optometry 2002; 73: 435-446.

6 Sanders DR, Vukich JA, Gaston M, Implantable Contact Lens in Treatment of Myopia Study Group. US Food and Drug Administration clinical trial of the implantable contact lens for moderate to high myopia. Ophthalmology 2003; 110: 255-266.

7 Lackner B, Pieh S, Schmidinger G, Hanselmayer G, DejacoRuhswurm I, Funovics MA et al. Outcome after treatment of ametropia with implantable contact lenses. Ophthalmology 2003; 110: 2153-2161.

8 Sanders DR, Doney K, Poco M, ICL in Treatment of Myopia Study Group. United States Food and Drug Administration clinical trial of the Implantable Collamer Lens (ICL) for moderate to high myopia: three-year follow-up. Ophthalmology 2004; 111: 1683-1692.

9 Pineda-Fernandez A, Jaramillo J, Vargas J, Jaramillo M, Jaramillo J, Galindez A. Phakic posterior chamber intraocular lens for high myopia. J Cataract Refract Surg 2004; 30: 2277-2283.

10 Kamiya K, Shimizu K, Igarashi A, Hikita F, Komatsu M. Four-year follow-up of posterior chamber intraocular lens implantation for moderate to high myopia. Arch Opthalmol; in press.

11 Sanders DR, Schneider D, Martin R, Brown D, Dulaney D, Vukich $\mathrm{J}$ et al. Toric Implantable Collamer Lens for moderate to high myopic astigmatism. Ophthalmology 2007; 114: 54-61.

12 Schallhorn S, Tanzer D, Sanders DR, Sanders ML. Randomized prospective comparison of visian toric implantable collamer lens and conventional photorefractive keratectomy for moderate to high myopic astigmatism. J Refract Surg 2007; 23: 853-867.

13 Kamiya K, Shimizu K, Igarashi A, Komatsu M. Comparison of Collamer toric contact lens implantation and wavefrontguided laser in situ keratomileusis for high myopic astigmatism. J Cataract Refract Surg 2008; 34: 1687-1693.

14 Kamiya K, Shimizu K, Ando W, Asato Y, Fujisawa T. Phakic toric Implantable Collamer Lens implantation for the correction of high myopic astigmatism in eyes with keratoconus. J Refract Surg 2008; 24: 840-842.
15 Freedman KA, Brown SM, Mathews SM, Young RS. Pupil size and the ablation zone in laser refractive surgery: considerations based on geometric optics. J Cataract Refract Surg 2003; 29: 1924-1931.

16 Gonvers M, Bornet C, Othenin-Girard P. Implantable contact lens for moderate to high myopia: relationship of vaulting to cataract formation. J Cataract Refract Surg 2003; 29: 918-924.

17 Gonvers M, Othenin-Girard P, Bornet C, Sickenberg M. Implantable contact lens for moderate to high myopia: short-term follow-up of 2 models. J Cataract Refract Surg 2001; 27: 380-388.

18 Kamiya K, Shimizu K, Komatsu M. Factors affecting the vaulting after Implantable Collamer Lens implantation. J Refract Surg 2009; 25(3): 259-264.

19 Keuch RJ, Bleckmann H. Pupil diameter changes and reaction after posterior chamber phakic intraocular lens implantation. J Cataract Refract Surg 2002; 28: 2170-2172.

20 Chun YS, Park IK, Lee HI, Lee JH, Kim JC. Iris and trabecular meshwork pigment changes after posterior chamber phakic intraocular lens implantation. J Cataract Refract Surg 2006; 32: 1452-1458.

21 Petternel V, Köppl CM, Dejaco-Ruhswurm I, Findl O, Skorpik C, Drexler W. Effect of accommodation and pupil size on the movement of a posterior chamber lens in the phakic eye. Ophthalmology 2004; 111: 325-331.

22 Baumeister M, Buhren J, Kohnen T. Position of anglesupported, iris-fixated, and ciliary sulcus-implanted myopic phakic intraocular lenses evaluated by Scheimpflug photography. Am J Ophthalmol 2004; 138: 723-731.

23 Bechmann M, Ullrich S, Thiel MJ, Kenyon KR, Ludwig K. Imaging of posterior chamber phakic intraocular lens by optical coherence tomography. J Cataract Refract Surg 2002; 28: 360-363.

24 Baikoff G. Anterior segment OCT and phakic intraocular lenses: a perspective. J Cataract Refract Surg 2006; 32: 1827-1835.

25 Birren JE, Casperson RC, Botwinick J. Age changes in pupil size. J Gerontol 1955; 5: 216-225.

26 Kadlecova V, Peleska M, Vasko A. Dependence on age of diameter of the pupil in the dark. Nature 1958; 82: 1520-1521.

27 Seitz R. The dependence on age of the dilation of the dark-adapted pupil. Klin Mbl Augenheilk 1957; 131: 48-56.

28 Said FS, Sawires WS. Age dependence of changes in pupil diameter in the dark. Optica Acta 1972; 19: 359-361.

29 Korczyn AD, Laor N, Nemet P. Sympathetic pupillary tone in old age. Arch Ophthalmol 1976; 94: 1905-1906.

30 Lowenfeld IE. Pupillary changes related to age. In: Thompson HS (ed.). Topics in Neuro-Ophthalmology. Williams \& Wilkins: Baltimore, 1979 pp 124-150.

31 Koch DD, Samuelson SW, Haft EA, Merin LM. Pupillary size and responsiveness. Ophthalmology 1991; 98: 1030-1035.

32 Camellin M, Gambino F, Casaro S. Measurement of the spatial shift of the pupil center. J Cataract Refract Surg 2005; 31: 1719-1721.

33 Fry GA. The relation of pupil size to accommodation and convergence. Am J Optom 1945; 22: 451-465.

34 Marg E, Morgan MW. The pupillary near reflex: the relation of pupillary diameter to accommodation and various components of convergence. Am J Optom 1949; 26: 183-198.

35 Hess EH. Pupillometrics: a method of studying mental, emotional, and sensory processes. In: Greenfield NS, Sturnbach RA (eds). Handbook of Psychophysiology. Holt, Reinhardt and Winston: New York, 1972 pp 491-534. 
36 Wyatt HJ, Musselman JF. Pupillary light reflex in humans: evidence for an unbalanced pathway from nasal retina, and for signal cancellation in brain-stem. Vision Res 1981; 21: 513-525.

37 Winn B, Whitaker D, Elliott DB, Phillips NJ. Factors affecting light-adapted pupil size in normal human subjects. Invest Ophthalmol Vis Sci 1994; 35: 1132-1137.

38 Pérez-Santonja JJ, Alió JL, Jiménez-Alfaro I, Zato MA. Surgical correction of severe myopia with an angle supported phakic intraocular lens. J Cataract Refract Surg 2000; 26: 1288-1302.

39 Alió JL, de la Hoz F, Pérez-Santonja JJ, Ruiz-Moreno M, Quesada JA. Phakic anterior chamber lenses for the correction of myopia; a 7-year cumulative analysis of complications in 263 cases. Ophthalmology 1999; 106: 458-466.

40 Jiménez-Alfaro I, Benítez del Castillo JM, García-Feijoó J, Gil de Bernabé JG, Serrano de La Iglesia JM. Safety of posterior chamber phakic intraocular lenses for the correction of high myopia: anterior segment changes after posterior chamber phakic intraocular lens implantation. Ophthalmology 2001; 108: 90-99.

41 Lackner B, Pieh S, Schmidinger G, Simader C, Franz C, Dejaco-Ruhswurm I et al. Long-term results of implantation of phakic posterior chamber intraocular lenses. J Cataract Refract Surg 2004; 30: 2269-2276. 